\title{
DESC de médecine d'urgence, quel bilan au bout de dix ans : bien, mais doit mieux faire
}

\author{
Assessment of French Emergency Medicine Training after Ten Years: Good, but could be Better
}

\author{
J. Levraut \\ (C) SFMU et Lavoisier SAS 2015
}

La médecine d'urgence est une discipline médicale que bien des pays dans le monde ont reconnue comme une spécialité à part entière depuis plusieurs dizaines d'années. Au cours de sa formation, le futur médecin urgentiste doit acquérir des compétences en termes de savoir, de savoir-faire et de savoir-être. L'urgentiste n'est pas seulement un médecin ayant des connaissances solides sur les pathologies d'urgence et leur traitement, mais il doit également être capable de réaliser un triage de qualité, émettre des hypothèses diagnostiques face à des motifs de recours, évaluer le degré de gravité et quantifier le risque face à une pathologie aiguë. Ceci l'oblige à avoir une vision transversale des parcours de soins et il devient ainsi un acteur incontournable de notre système de santé.

Cette acquisition de compétences spécifiques repose sur une formation qui a été initiée en France par la mise en place de diplômes d'université puis en 1986 par la création de la capacité d'aide médicale urgente (CAMU), qui visait essentiellement à former les médecins exerçant en SAMU. Devant le rapprochement des concepts pré- et intrahospitaliers au milieu des années 1990, la CAMU était remplacée en 1998 par la capacité de médecine d'urgence (CMU). Celle-ci reposait sur une formation théorique de 160 heures et une formation pratique de 800 heures de stage réparties sur deux ans. Face à la demande croissante d'accès à cette discipline et à la nécessité de renforcer la formation pratique par compagnonnage, le diplôme d'études spécialisées complémentaires (DESC) de médecine d'urgence a été créé en 2004. Il s'agit d'un DESC de type 1, c'est-à-dire qu'il est non qualifiant et ne donne actuellement pas une reconnaissance ordinale de la spécialisation.
Dans ce numéro des Annales Françaises de Médecine d'Urgence, Dehours et al. [1] rapportent le ressenti des urgentistes quant à leur formation, évalué par un questionnaire adressé aux médecins urgentistes formés dans la région Sud-Ouest. Il ressort de cette enquête que les médecins sont globalement satisfaits de leur formation. Ils estiment cependant manquer d'apprentissage de gestes techniques ainsi que de formation dans divers domaines tels que la pédiatrie, la régulation médicale ou la petite traumatologie. L'ensemble des étudiants auraient en outre souhaité bénéficier de plus de séances de simulation, particulièrement bien adaptée pour l'apprentissage du savoir-faire et du savoir-être.

Seule une formation plus longue avec un compagnonnage renforcé permettrait de satisfaire correctement les besoins de formation que seul un internat de spécialisation permettrait de garantir. Il est donc impératif que le DESC soit rapidement remplacé par la création du DES de médecine d'urgence, seule façon de satisfaire pleinement les besoins de formation, de garantir les exigences de recrutement médical et de favoriser le développement académique de la discipline. Ce n'est qu'à ce prix-là que la médecine d'urgence acquerra définitivement ses titres de noblesse et s'imposera à tous comme une spécialité médicale à part entière.

\section{Référence}

1. Dehours E, Moustafa F, Jaudet G, Lauque D (2015) Que pensent les urgentistes de la région Sud-Ouest de leur formation par le DESC de médecine d'urgence, dix ans après sa création ? Ann Fr Med Urg 5:144-9

\footnotetext{
J. Levraut $(\square)$

Pôle Urgences SAMU SMUR - CHU de Nice

e-mail : levraut.j@chu-nice.fr
}

Hôpital Saint Roch, 5 rue Pierre Dévoluy, F-06006 Nice cedex 1 